HEALTHCARE DELIVERY

\title{
Burkitt's lymphoma: The prevalence of HIV/AIDS and the outcome of treatment
}

\author{
P B Hesseling, ${ }^{1}$ MD; F Kouya, ${ }^{2} \mathrm{MD}$; E Katayi,${ }^{2} \mathrm{MD}$; G Mbah, ${ }^{2} \mathrm{RN} ;$ P Wharin, ${ }^{3} \mathrm{MD}$ \\ ${ }^{1}$ Department of Paediatrics and Child Health, Tygerberg Children's Hospital and Faculty of Medicine and Health Sciences, Stellenbosch University, \\ South Africa \\ ${ }^{2}$ Cameroon Baptist Convention Health Board, Bamenda, Cameroon \\ ${ }^{3}$ Beryl Thyer Memorial Africa Trust, Warkton, UK
}

Corresponding author: P B Hesseling (pbh@sun.ac.za)

\begin{abstract}
The prevalence of HIV in Burkitt's lymphoma (BL) patients and the outcome of treatment in Cameroon were unknown. Records of all patients diagnosed with BL at three Cameroon Baptist Convention hospitals were reviewed to ascertain the recorded HIV status and outcome of treatment. Of 979 patients diagnosed with BL, 717 were tested for HIV and 11 (1.5\%) were HIV-positive. Three of eight patients treated with both cyclophosphamide (CPM)-based chemotherapy and antiretrovirals were alive at 62, 96 and 111 months, respectively. The HIV rate was comparable to that of $1 \%$ for the general population of children aged $<15$ years. Low-cost high-frequency CPM was the only available treatment option for BL and was associated with $37.5 \%$ long-term survival in a resource-limited setting.
\end{abstract}

S Afr Med J 2018;108(2):84-85. DOI:10.7196/SAMJ.2018.v108i2.12441

The population of Cameroon in 2014 was 22773 014, of whom $\sim 40 \%$ (9 109 205) were aged $<15$ years. It was estimated that 94000 of these children (1\%) were infected with HIV, and only $11 \%$ of these received antiretroviral (ARV) therapy. ${ }^{[1]}$ In 2013, the prevalence of HIV infection in pregnant women in the north-west and south-west regions was $4.6 \%$ and $6.8 \%$, respectively, and the mother-to-child transmission rate in 18 -month-old breastfed infants was $25 \% .^{[1]}$ Children diagnosed with Burkitt's lymphoma (BL) between 2003 and 2015 at Banso and Mbingo Baptist hospitals in the north-west and Mutengene Baptist Hospital in the south-west were all treated with cyclophosphamide-based chemotherapy regimens, with an overall long-term survival rate of $55 \%{ }^{[2]}$

\section{Objective}

To record the prevalence of HIV and the outcome of treatment in a large cohort of patients with BL.

\section{Methods}

Our database is a Pediatric Oncology Network Database (POND) cancer registry for the period 2003 - 2013. The number of patients in whom enzyme-linked immunosorbent assay was positive for $\mathrm{HIV}$, the CD4+ count when available, and whether ARV treatment was given or not were extracted from the database. The diagnosis of BL was based on a fine-needle tumour aspirate, a bone marrow aspirate, a cytological cerebrospinal fluid examination and an abdominal ultrasound scan. The long-term outcome was established by personal follow-up of every HIV-positive patient or their families. Chemotherapy induction treatment consisted of cyclophosphamide (CPM) $40 \mathrm{mg} / \mathrm{kg}$ on days 1,8 and 15 , followed by consolidation chemotherapy with $1-3$ more doses of CPM with or without intravenous (IV) vincristine $1.5 \mathrm{mg} / \mathrm{m}^{2}$ and IV methotrexate $1.0 \mathrm{~g} / \mathrm{m}^{2}$ for advanced or non-responsive disease. The duration of chemotherapy was 2 months. Rescue treatment of early relapses consisted of 3 weekly pulses of CPM and vincristine, and relapses beyond 1 year were treated in full again. ${ }^{[3]}$ ARV treatment was not freely available in the early years of the study. More recent treatment always consisted of three drugs, based on World Health Organization guidelines and the national protocol. Examples of first-line treatment are as follows: age $<3$ years - abacavir + lamivudine + lopinavir/ ritonavir; age $3-10$ years and adolescents weighing $<35 \mathrm{~kg}-$ abacavir + lamivudine + efavirenz; and age $11-19$ years or weight $>35 \mathrm{~kg}$ - tenofovir + lamivudine (or emtricitabine) + efavirenz.

Ethical approval for the BL treatment protocols was obtained from the Institutional Review Board of the Cameroon Baptist Convention Health Board.

\section{Results}

Of 979 patients diagnosed with BL, 717 (73.2\%) were tested for HIV and $11(1.5 \%)$ tested positive. Their ages ranged from 2 to 13 years (median 7 years). The age at diagnosis of BL, St Jude stage of the $\mathrm{BL}, \mathrm{CD} 4+$ count if done, ARV treatment given, current status and duration of long-term survival are listed in Table 1. On 30 October 2016, three of eight patients (37.5\%) who had received the recommended ARV treatment and were still on ARV maintenance treatment were long-term survivors at 111, 96 and 62 months, respectively. Three patients who were not treated with ARVs died within 3-15 months, and five patients treated with ARVs died within 3 - 39 months after the onset of treatment from progressive disease. There was no chemotherapy-related death. The CD4+ count at diagnosis was recorded in only four patients.

\section{Discussion}

Children with HIV have an increased risk of developing malignancies, and BL accounted for $21 \%$ of malignancies in HIV-positive children in South Africa, with a projected cure rate of $54.6 \%$ at 60 months using modern chemotherapy. ${ }^{[4]}$ Modern chemotherapy for BL has a potential cure rate of $>90 \%$ in HIV-negative patients, ${ }^{[5]}$ but at considerable cost and the risk of significant morbidity. Our BL 


\section{Table 1. Patient details and outcome}

\begin{tabular}{|c|c|c|c|c|c|c|}
\hline Patient no. & Age (years) & St Jude stage & $\begin{array}{l}\text { CD4+ count } \\
(\text { cells } / \mu \mathrm{L})\end{array}$ & ARV treatment & Current status & $\begin{array}{l}\text { Length of } \\
\text { survival } \\
\text { (months) }\end{array}$ \\
\hline 1 & 5 & III & No record & No & Dead & 15 \\
\hline 2 & 12 & III & 145 & Yes & Dead & 6 \\
\hline 3 & 7 & III & No record & Yes & Alive & 96 \\
\hline 4 & 3 & III & 559 & Yes & Alive & 111 \\
\hline 5 & 9 & III & No record & No & Dead & 3 \\
\hline 6 & 2 & III & No record & Yes & Alive & 62 \\
\hline 7 & 4 & II & No record & Yes & Dead & 4 \\
\hline 8 & 3 & III & No record & No & Dead & 3 \\
\hline 9 & 8 & III & No record & Yes & Dead & 39 \\
\hline 10 & 12 & IV & 80 & Yes & Dead & 39 \\
\hline 11 & 13 & III & 30 & Yes & Dead & 3 \\
\hline
\end{tabular}

patients were treated with chemotherapy of much lower intensity because of limited access to drugs and supportive care. ${ }^{[6]}$ Long-term survival of 5 - 9 years in three of eight patients (37.5\%) who received both chemotherapy and ARVs is encouraging. The documented HIV prevalence rate of $1.5 \%$ in this large cohort of BL patients is comparable to the $1 \%$ HIV prevalence rate for the general population of children in Cameroon.

\section{Conclusion}

HIV-positive children with BL are best treated with a contextappropriate chemotherapy regimen.

Acknowledgements. Poster presentation at the 48th congress of the International Society of Paediatric Oncology (SIOP), Dublin, October 2016. Author contributions. PBH: Principal investigator, FK: physician in charge of the children's cancer service, EK: physician in charge of the HIV service of the Cameroon Baptist Convention Health Board, GM: research assistant responsible for the cancer registry and data co-ordination, $\mathrm{PW}$ : involved in planning and follow-up.
Funding. Funding was received from the Beryl Thyer Memorial Africa Trust (UK), World Child Cancer (UK) and the Swiss Cancer League.

\section{Conflicts of interest. None}

1. Joint United Nations Programme on HIV/AIDS (UNAIDS). 2015 progress report on the Global Plan towards the elimination of new HIV infections among children and keeping their mothers alive. http.// www.unaids.org/sites/default/files/media_asset/JC2774_2015ProgressReport_GlobalPlan_en Pdf (accessed 30 December 2017)

2. Hesseling P, Kouya F, Mbah G, et al. Burkitt lymphoma: Long term outcome in 738 children treated with cyclophosphamide-based protocols in rural Cameroon from 2003 to 2013 (abstract O-043). Pediatr Blood Cancer 2015:62(S4):S156. https://doi.org/10.1002/pbc.25715

3. Hesseling PB, Njume E, Kouya F, et al. The Cameroon 2008 Burkitt lymphoma protocol: Improved survival with treatment adapted to disease stage and response to treatment. Pediatr Hematol Oncol 2012;29(2):119-129. https://doi.org/10.3109/08880018.2011.64488

4. Davidson A, Wainwright RD, Stones DK, et al. Malignancies in South African children with HIV. J Pediatr Hematol Oncol 2014;36(2):111-117. https://doi.org/10.1097/MPH.0b013e31829cdd49

5. Patte C, Auperin A, Michon I, et al. The Societe Francaise d'Oncologie Pediatrique LMB 89 Protocol: Highly effective multi-agent chemotherapy tailored to the tumour burden and initial response in 561 unselected children with B-cell lymphoma and L3 leukaemia. Blood 2001;97(11):3370-3379. https:// doi.org/10.1182/blood.V97.11.3370

6. Hesseling P, Israels T, Harif M, Chantada G, Molyneux E. Practical recommendations for the management of children with endemic Burkitt lymphoma in a resource limited setting. Pediatr Blood Cancer 2013;60(3):357-362. https://doi.org/10.1002/pbc.24407

Accepted 5 September 2017. 